\title{
Phase separation and emergence of order in a one-dimensional system of active particles
}

\author{
Lucas Barberis ${ }^{1,2}$ and Fernando Peruani ${ }^{1}$ \\ ${ }^{1}$ Université Nice Sophia Antipolis, Laboratoire J.A. Dieudonné, \\ UMR 7351 CNRS, Parc Valrose, F-06108 Nice Cedex 02, France \\ ${ }^{2}$ Intituto de Física Enrique Gaviola, FaMAF, CONICET, UNC, \\ Haya de la Torre s/n, Ciudad Universitaria, X5000HUA, Córdoba, Argentina
}

\begin{abstract}
We study numerically a one-dimensional systems of self-propelled particles, where the state of the particles is given by their moving direction (left or right), which is encoded by a spin-like variable, and their position. Particles interact by short-ranged, spring-like attractive forces and do not possess spin-spin interactions (i.e. velocity alignment). Newton's third law is broken in this model by assuming an asymmetric interaction range that is larger in the direction of the moving direction of the particle. We show that in this nonequilibrium system, due to the absence of the action-reaction symmetry, there exists an intimate link between phase separation and the formation of highly-coherent, spatially localized, moving flocks (i.e. collective motion). More specifically, we prove the existence of two fundamentally different types of active phase separation, which we refer to as neutral (NPS) and polar (PPS) phase separation. Furthermore, we indicate that NPS is subdivided in two classes with distinct critical exponents. These results are of key importance to understand that in Active Matter there exist several phase-separation classes and that the emergence of polar, self-organized patterns (i.e. flocks) does not require the presence of a velocity alignment.
\end{abstract}

\section{INTRODUCTION}

Complex, self-organized, collective motion patterns observed in nature, from birds to sheep $[1-6]$ and including those emerging in non-living active systems [7-10] are believed to require velocity-velocity interactions that are often referred to as velocity alignment. Such velocity alignment mechanism is at the core of the so-called Vicsek-like models [11] extensively used to study flocking patterns $[1,2,12-16]$. However, it has been recently suggested that self-organized patterns in active systems may emerge by alternative mechanisms [17-22].

In [22], we have introduced and analyzed a simple twodimensional active system where the self-propelled particles interact with those particles inside a field of view via an attractive force. By construction, this model does not possess velocity-velocity interactions and thus, there is no built-in microscopic interaction rule with either polar or apolar symmetry. And yet, we have proved that in this active model, polar and nematic (velocity) order spontaneously emerge in different areas of the parameter space in the absence of action-reaction symmetry [22, 23]. This is in sharp contrast with what it is observed in active systems with velocity alignment [11-13, 24-35], where the emergence of polar order requires a microscopic polar alignment rule [11] and nematic order, a microscopic nematic alignment rule [30, 32] (see also [42]). In short, the two-dimensional active model studied in [22] proves that order can emerge in the absence of velocity-velocity interactions, without built-in microscopic rules with a predefined symmetry, and as result of a dynamical process.

Here, we show that local order may also emerge in one-dimensional active systems without velocity-velocity interactions. In a one-dimensional system, the velocity

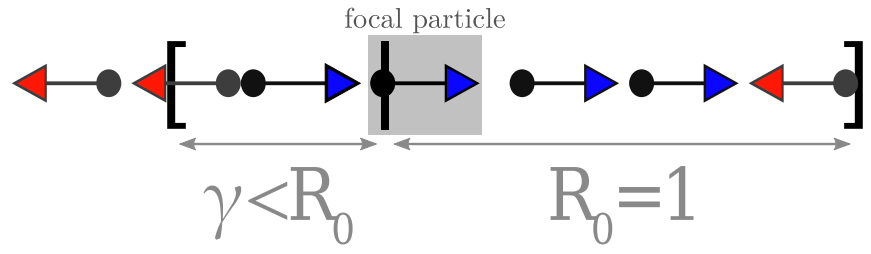

FIG. 1: Breaking Newton's third law. Scheme illustrates the asymmetric interacting neighborhood of the self-propelled particles: the focal particle interacts (via an Harmonic potential with cut-off) with all particles that are located up to a distance $R_{0}=1$ in front of the particle - it is, in the direction indicated by the spin of the focal particle, denoted by the blue arrow - and with those particles located up to a distance $\gamma<R_{0}$ in the opposite direction. See Eq. (1) and the description below it.

or rather the moving direction of the particles is either to the left or to the right, implying that can be represented by a spin variable $S$, with $S=+1$ or $S=-1$. Thus, we can assign to each particle a spin, e.g. $S_{i}$ for particle $i$ and define a dynamics by which the state of the particle is given by its position, $x_{i}$, and its spin, $S_{i}$; for us, such dynamics consists of short-ranged, springlike attractive forces. In spin models such as in the classical $[36,37]$ or active Ising model [16], the emergence of ordered or magnetized domains - in the sense of regions where $\left|\sum_{j} S_{j}\right|>0$ - results from one of the following (equivalent) mechanisms: i) the local minimization of some spin-spin interaction potential such as e.g. $H=-J \sum_{i, j} S_{i} S_{j}[36]$, ii) the use of a (local) spin flipping rate such as $\exp \left(S \sum_{j} S_{j} / A\right)$, where $S$ is the spin that is probed and $A$ depends on the local configuration [16], or iii) the implementation of a local (stochastic 
or deterministic) majority rules based on the local value of $\sum_{j} S_{j}[14,15]$. Our study shows that in the absence of such spin-spin interaction potential or spin flipping rate (dependent on the local magnetization), it is still possible to create (local) order by breaking the actionreaction symmetry. Here, order emergence results from a dynamical process. Furthermore, we indicate that existence of an intimate connection between phase separation and the emergence of highly-coherent, spatially localized, moving flocks (i.e. collective motion). Notably, we show that there exist two fundamentally different types of active phase separation, which we call as neutral (NPS) and polar (PPS) phase separation; cf. [38, 39]. In addition, we indicate that NPS is subdivided in two classes with distinct critical exponents. The obtained results are of key importance to understand the emergence of self-organized, ordered structures and phase separation in out-of-equilibrium systems such as active systems.

\section{MODEL DEFINITION}

We consider a one-dimensional system of size $L$ with periodic boundary conditions, where the state of the $i$-th particle is given by its "spin" $S_{i}$ - which refers to the moving direction of the particle - and its position $x_{i}$, where $S_{i} \in\{-1,+1\}, x_{i} \in(0, L]$, and $i=1 \ldots N$. The equation of motion of the $i$-th particle - that interact with other particles exclusively by an attractive force is given by:

$$
\dot{x}_{i}=v_{0} S_{i}+K_{0} \sum_{\Omega\left(x_{i}, S_{i}\right)}\left(x_{j}-x_{i}\right)+\sqrt{2 D} \xi(t),
$$

where $v_{0}$ is the active speed, $K_{0}^{-1}$ has units of time, $D$ is a diffusion constant, and $\xi$ is a delta-correlated noise. One essential element of the model is given by the set $\Omega\left(x_{i}, S_{i}\right)$ that contains all neighbors of $i$-th particle. This set is defined for $S_{i}=1$ by all particles whose positions are in the interval $\left[x_{i}-\gamma, x_{i}+R_{0}\right]$. For $S_{i}=-1$, the corresponding interval is $\left[x_{i}-R_{0}, x_{i}+\gamma\right]$; see Fig. 1. Both, $R_{0}$ and $\gamma$ are positive constants and such that $\gamma \leq R_{0}$. Note that the intervals have to be properly defined using periodic boundary condition, i.e. if the interval is $[a, b]$, then $a \leftarrow(a+L) \bmod L$ and $b \leftarrow(b+L) \bmod L$. We stress that when $\gamma=R_{0}$, the interactions are reciprocal and Newton's third law applies, while for $\gamma<R_{0}$ the action-reaction symmetry is not longer present.

Finally, the dynamics of the spin $S_{i}$ is simply:

$$
S_{i} \stackrel{\alpha}{\longrightarrow}-S_{i}
$$

where $\alpha$ is considered a function of the number $n_{i}$ of neighbors of particle $i$ :

$$
\alpha\left[n_{i}\right]=\mu e^{-\beta n_{i}},
$$

where $\mu$ (in inverse time units) and $\beta$ (nondimensionalized) constants and $n_{i}$ the cardinal number of the set $\Omega\left(x_{i}, S_{i}\right)$. For $\beta=0$, Eq. (2) defines a standard Poisson process. In the following, the reported numerical results correspond to global density $\rho_{0}=N / L=1$ and without loss of generality of fix $R_{0}, K_{0}$, and $v_{0}$ to 1 , and $D=10^{-2} / 2$.

\section{LESSONS FROM THE DETERMINISTIC PARTICLE DYNAMICS}

To understand the deterministic dynamics of the system, let us consider $\alpha=0$ and $D=0$ and focus on the dynamics of a cluster of $M$ particles where each particle interact with the other $M-1$ particles at any given time. The resulting dynamical system is such that the equation of motion of the $i$-th particle is given by: $\dot{x}_{i}=v_{0} S_{i}+K_{0} \sum_{i}\left(x_{i}-x_{k}\right)$. For any particle $i$, the spin $S_{i}$ is given by the initial condition and remains unchanged, but the particle position $x_{i}$ evolves in time. Under these conditions, it is evident that:

$$
\frac{1}{M} \sum_{k=1}^{M} \dot{x}_{i}=\frac{v_{0}}{M} \sum_{i=1}^{M} S_{i}=v_{0} m_{M}=V,
$$

where $m_{M}=\left[\sum_{i=1}^{M} S_{i}\right] / M$ is the (average) "magnetization" of the cluster and $V$ the velocity of its center of mass. By placing ourselves in a frame of reference that moves at velocity $V$, we define $y_{i}=x_{i}-V t$ and thus $\sum_{i} \dot{y}_{i}=0$, implying that $\sum_{i} y_{i}=Y_{0}$ with $Y_{0}$ a constant such that $Y=Y_{0} / M$ represents the position of the center of mass at $t=0$. By defining $z_{i}=x_{i}-V t-Y$, whose evolution is given by

$$
\dot{z}_{i}=v_{0} S_{i}-V+K_{0} \sum_{j}\left(z_{j}-z_{i}\right),
$$

we obtain a system where $\sum_{i} \dot{z}_{i}=0$ and $\sum_{i} z_{i}=0$, i.e. the center of mass is located at the origin at all times. The main advantage of this is that the steady state of Eq. (5) can be easily computed as:

$$
z_{i}=\frac{v_{0}}{K_{0} M}\left(S_{i}-m_{M}\right)
$$

Since $S_{i}$ adopts only two values, either +1 or -1 , then the position of the left most particle in the cluster is $z_{+}=\frac{v_{0}}{K_{0} M}\left(1-m_{M}\right)$ and the right most position $z_{-}=$ $\frac{v_{0}}{K_{0} M}\left(-1-m_{M}\right)$, assuming that there is at least one particle with spin +1 and one particle with spin -1 . Thus, the "width" $W$ of the cluster is:

$$
W=z_{+}-z_{-}=\frac{2 v_{0}}{K_{0} M} .
$$

Note that the cluster width $W$ does not depend on the magnetization $m_{M}$, but only on the number of particles 
(a)

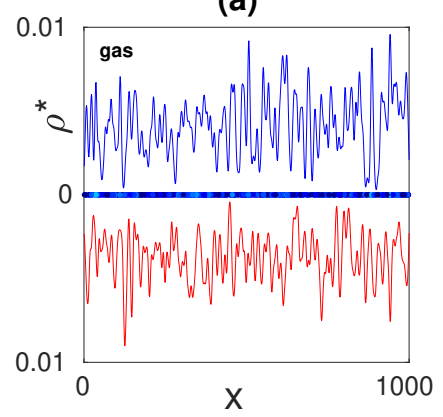

(b)

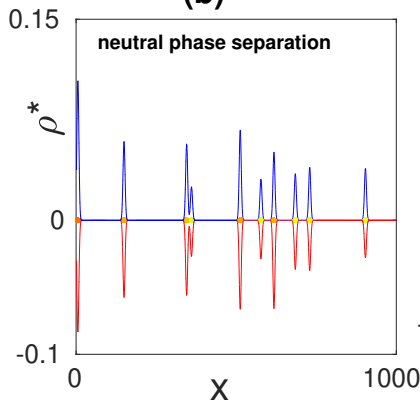

(c)

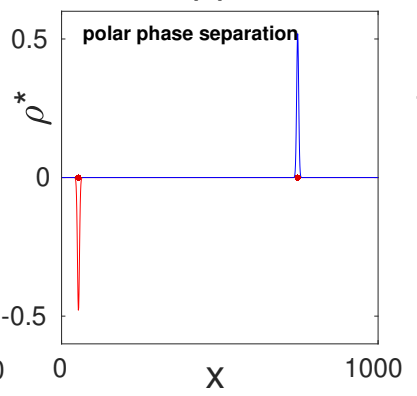

(d)

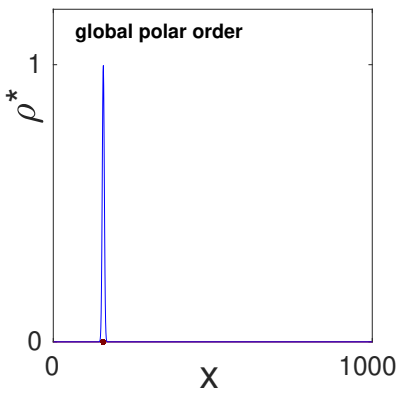

FIG. 2: Self-organized patterns. Active particles self-organize into various phases depending on model parameters. The possible phases are: (a) disordered gas, (b) neutral phase separation (NPS), (c) polar phase separation (PPS), or (d) the emergence of global order in finite size systems. Blue and red lines represent the (smooth) density of particle with $S=+1\left(\rho^{+}\right)$and $S=-1$ $\left(\rho^{-}\right)$spins, respectively. The smoothing is performed as follows: $\rho^{+}(x, t)=\sum_{i=1}^{N} \delta\left(S_{i},+1\right) \exp \left(x-x_{i}\right) / 20$, and similar for $\rho^{-}$.

$M$ it contains. We insist that Eq. (7) only applies when $\left|m_{M}\right|<1$. For $\left|m_{M}\right|=1$, the width vanishes. In summary, the velocity of a cluster is given by the average magnetization $m_{M}$ and the cluster width is a decreasing function of the cluster mass $M$ for $\left|m_{M}\right|<1$ and zero otherwise.

There is an important lesson to learn from these simple calculations when we include the spin-flip dynamics and implement a finite range of interaction $R_{0}$ and consequently also $\gamma$. A first evident observation is that for large values of $D$ and low density values, the system exhibits a disordered, gas phase as shown in Fig.2(a). On the other hand, for small $D$ values and $\beta=0$, which ensures that all clusters are in average neutral (i.e. clusters cannot exhibit persistent magnetization), the system phase separates. The phase separation process can be understood as follows. The biggest cluster width $W$ corresponds to $M=2$, i.e. $v_{0} / K_{0}$. If $\gamma>v_{0} / K_{0}$, whenever two particles get at a distance $\left|x_{i}-x_{j}\right|<\gamma$, they form a "bond", which for small enough $D$ values is long-lived. With time, this dimer will encounter other clusters and fuse with them: in short, the system phase separates. On the other hand, for $\gamma<v_{0} / K_{0}$ and in fact for any arbitrary small $\gamma$, the formation of clusters requires a random density fluctuation such that in an interval of width $\gamma$ we find $M>2 v_{0} /\left(K_{0} \gamma\right)$ particles. The time to observe the formation of such a cluster determines the nucleation time of the process. Once many clusters emerge, the clusters start fusing with each other and the system phase separates.

For $\beta>0, \alpha[n]$ becomes a function of the number of particles $n$ in the interaction neighborhood and the system displays a much more complex dynamics. By suppressing the flipping rate in high density regions (as consequence of $\beta>0$ ), polar arrangements of particles are stabilized in the sense that if a density fluctuation results in a number of particles $n>\beta^{-1}$ with the same spin in a range $R_{0}$, these particles will keep on moving in the same direction; this is true as long as $K_{0}$ and $D$ are such that allow them to stay close to each other. This does not prevent these particles to find a cluster of particles moving in the opposite direction. If this occurs, they will form a cluster where in general the magnitude of the average magnetization will be smaller than 1 , and due to the suppression of spin flips (since $\beta>0$ ), their magnetization, though very small, is persistent, in the sense that it remains constant until meeting a cluster or particle as the cluster moves ballistically across the system. Thus, the transport properties of these clusters are thus very different from the ones that emerge when $\alpha$ is a constant (see below). For small values of $\gamma$ and large enough values of $D$, configurations involving two clusters of opposite magnetization become unstable. In this scenario, we observe that polar cluster prevails and start fusing with other clusters of the same magnetization, which leads to the emergence of polar (or magnetized) clusters containing a macroscopic fraction of the particles in the system; see Fig. 2(c). In short, we observe that particles selforganize into a single flock moving to the right and a single flock moving to the left, with particle motion becoming asymptotically ballistic; see Fig. 2(c). In finite systems, it often observed the nucleation of a single polar cluster that quickly absorbes all particles in the system, leading to the emergence of global polar order; see Fig. $2(d)$. However, we insist that the emergence of a single flock can only occur in finite size systems.

Below we provide more details on the physics of this system and organize the discussion in two sections: "Reciprocal interaction" and "In the absence of actionreaction symmetry". 


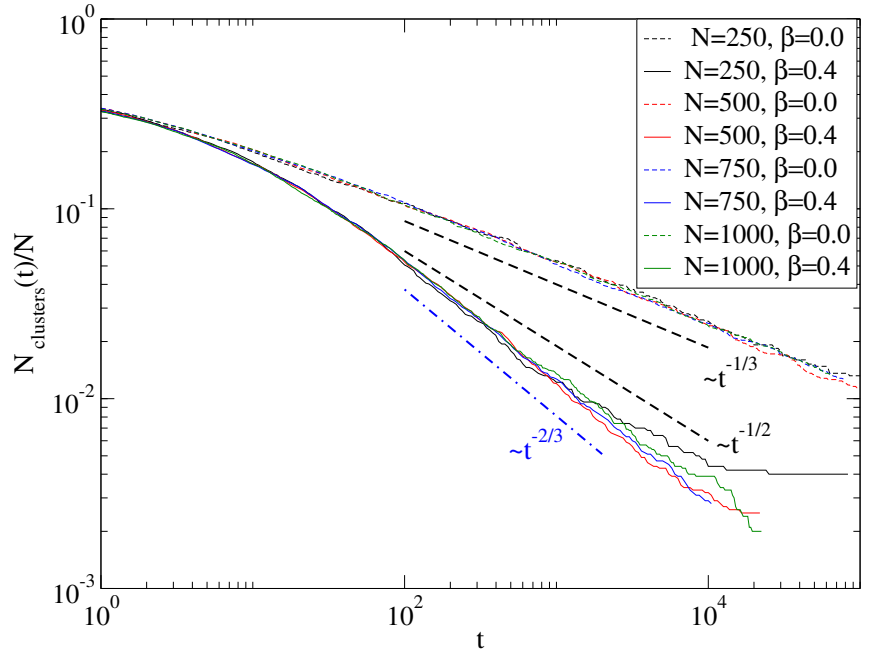

FIG. 3: The two classes of neutral phase separation (NPS). The number of clusters $N_{\text {clusters }}$ normalized by the number of particles in the system $N$ as function of time. For $\beta=0$, clusters display diffusive motion and we observe that asymptotically $N_{\text {clusters }} \propto t^{-1 / 3}$. For $\beta>0$, specifically $\beta=0.4$, cluster of large sizes exhibit ballistic motion, which leads to a different scaling: $N_{\text {clusters }} \propto t^{-2 / 3}$

\section{RECIPROCAL INTERACTIONS}

\section{two routes to neutral phase-separation}

When interactions are reciprocal, i.e. $\gamma=R_{0}$, Newton's third law applies. In this scenarios, the system is a disordered gas or it undergoes a "neutral phase separation" (NPS). We define NPS a phase separation where the growing, high-density regions - which we call clusters - exhibit a vanishing average magnetization as they increase their size over time. We use the term "polar phase separation" (PPS), as opposed to NPS, when the growing clusters display an average magnetization that does not decrease with cluster size: i.e. when the system phase separates in polar domains. For this to occur, interactions have to be nonreciprocal, see next section.

For reciprocal interactions, there exist two routes to phase-separate. For $\beta=0$, the spins forming a cluster are constantly flipping and clusters display diffusive motion. As result of this, phase-separation occurs via diffusion as in a classical Cahn-Hilliard process. On the other hand, for $\beta>0$ when cluster sizes are above $\beta^{-1}$, spin flipping is strongly suppressed and the motion of clusters becomes ballistic due to the random excess of spins +1 or -1 in those clusters when they formed; let us recall the magnetization of these cluster is persistent in between interaction with other clusters. Due to this, there is a "ballistic aggregation" of clusters. Differences in the asymptotic transport properties of the forming clusters lead to fundamentally different phase-separation classes.
These classes can be identified in term of the critical exponents that characterize the coarsening dynamics. The mean-field prediction for irreversible coarsening of diffusive clusters in $1 \mathrm{D}$ is $N_{\text {clusters }}(t) \propto t^{-1 / 3}$. For $\beta=0$ we expect diffusive clusters. Fig. (3) shows that simulations with $\beta=0$ are consistent with the mean-field prediction. For irreversible coarsening of ballistic clusters, the mean-field scaling is $N_{\text {clusters }}(t) \propto t^{-1 / 2}$. Simulations for reciprocal interactions and $\beta>0$, which lead to ballistic clusters, display a scaling consistent with a slightly different exponent: $-2 / 3$. This suggests that corrections to the mean-field prediction are required the understand the aggregation process in this limit, Fig. (3). However, the important observation - expected already at the meanfield level - is the existence of two very different (neutral) coarsening processes (and scalings), which are clearly illustrated in Fig. (3).

\section{IN THE ABSENCE OF ACTION-REACTION SYMMETRY}

\section{from phase separation to the emergence of order}

The physics of the problem is dramatically affected by breaking the action-reaction symmetry with the use of $\gamma<R_{0}$. The main effect of introducing nonreciprocal interactions is that now it is possible to weaken the interaction among clusters that are polarly oriented in opposite direction, while favoring the aggregation of clusters and particles with the same magnetization. This become evident in the limit of $\gamma \rightarrow 0$ and for $\beta>0$ : clusters of opposite magnetization speed up when they are approaching each other at a distance smaller than $R_{0}$. As soon as these clusters pass by each other, they do not interact anymore. In short, particles with spin $S=+1$ and $S=-1$ become almost independent of each other, except for spin-flip transitions in dilute areas of the space, which lead to an effective exchange between particle moving to the left and to the right. As result of this behavior, we observe first the formation of polar clusters, followed by the slow aggregation of polar clusters with the same magnetization, see Fig. 2(c). Consequently, in the system polar domains (or aggregates) emerge and phase separate. As we indicated above, we refer to this process polar phase separation.

It is worth stressing that with non-reciprocal interactions, it is possible to observe: a) a gas, b) that the system undergoes neutral phase separation (NPS), or c) that the system undergoes polar phase separation (PPS). In Fig. 4, we show the transition from polar to neutral phase separation using as control parameter $\gamma$ (as well as $\beta$ ). We characterize this transition by using two order parameters: $\phi=\frac{1}{N_{\text {clusters }}} \sum_{q=1}^{N_{\text {clusters }}}\left|m_{q}\right|$ - which is the average of the absolute value of the magnetization of individual clusters - and the asymptotic number of clusters, 
(a)

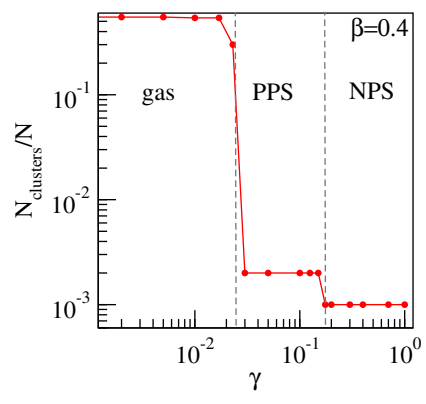

(c)

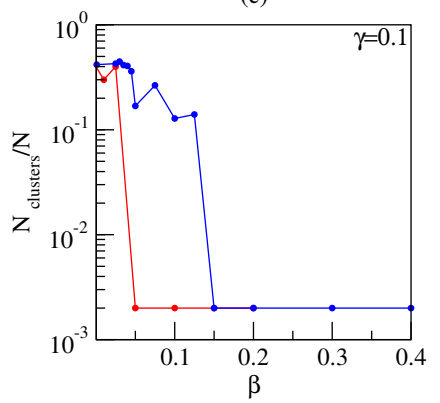

(b)

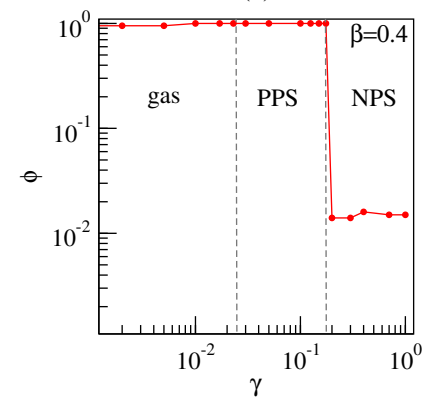

(d)

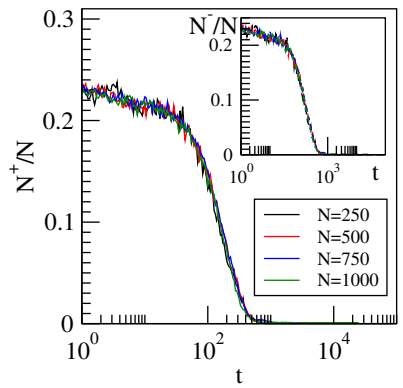

FIG. 4: Polar phase separation (PPS). a) The number of clusters $N_{\text {clusters }}$ normalized by the number of particles in the system $N$ as function of $\gamma$. b) The local order parameter $\phi$ as function of $\gamma$. In a) and b), $\beta=0.4$. c) $N_{\text {clusters }} / N$ as function of $\beta$ for $\gamma=0.1$. d) Temporal evolution of $N_{\text {clusters }}^{+}(t) / N$ and $N_{\text {clusters }}^{-}(t) / N$ (inset), where $N_{\text {clusters }}^{+}(t)$ and $N_{\text {clusters }}^{-}(t)$ refer to the number of clusters moving to the left and right, respectively. Note that in order to observe PPS, the actionreaction symmetry has to be broken, i.e. $\gamma<R_{0}=1$.

i.e. $N_{\text {clusters }} / N ; N_{\text {clusters }}$ without explicit dependency on $t$ refers to its asymptotic value: $N_{\text {clusters }}(t \rightarrow \infty)$. Note that if most clusters are neutral, then $\left|m_{q}\right| \ll 1$ and $\phi$ is small, while if most clusters are polar, then $\phi \rightarrow 1$. On the other hand, the information provided by $N_{\text {clusters }} / N$ allows us to determine whether the systems is phase-separated: $N_{\text {clusters }} / N \ll 1$ indicates that the system is phase separated, while $N_{\text {clusters }} / N \sim 1$ corresponds to a gas phase. Note that to distinguish between gas phases and neutral and polar phase separated phases, we need to use two order parameters, namely $\phi$ and $N_{\text {clusters }} / N$ : with $N_{\text {clusters }} / N \ll 1$ we know that the system is phase separated, while $\phi \sim 0$ indicates that the phase separated phase is neutral, while $\phi \sim 1$ that is polar. By varying $\gamma($ for $\beta>0$ ), the system transitions between neutral and polar phase-separated states as shown in Fig. 4; the phase diagram of the system is provided in Fig. 5. There exist important differences in terms of transport properties between between neutral and polar phase-separated states. During neutral phase separation, particles remain trapped inside neutral macroscopic aggregates. Asymptotically, neutral clusters fuse until the emergence of a single, macroscopic neutral cluster, which

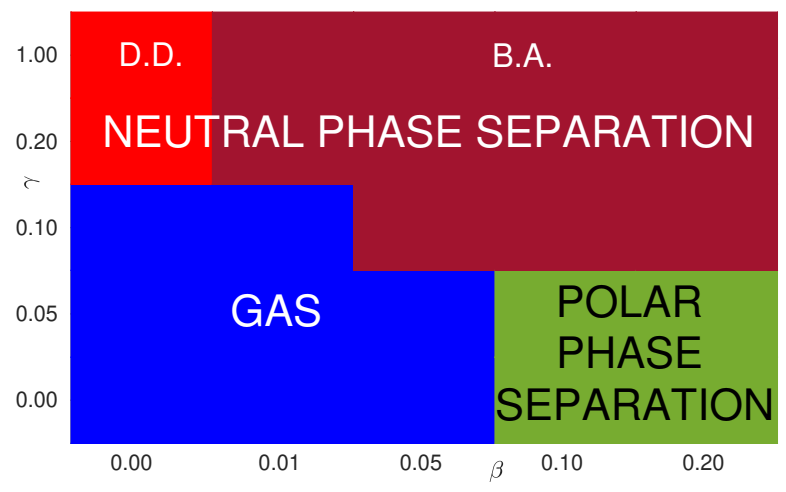

FIG. 5: The phase diagram of the system in the $\beta-\gamma$ parameter space. Gas occurs for small values of $\gamma$ and $\beta$. By decreasing the spin flipping rate by rising the value of $\beta$, the emerging clusters become polar and we observe polar phase separation (see text). As the interactions zone becomes symmetric, the magnetization of the emerging clusters tend to vanish (i.e. they become neutral). Here, as indicated in the text, we may observe diffusion dominated phase separation or ballistic aggregation.

is an immobile (in the thermodynamic limit) giant cluster [43]. During polar phase separation state, contrary to this, clusters keep on moving ballistically at the active speed $v_{0}$. And asymptotically, particles self-organize into two (spatially localized) macroscopic flocks moving in opposite directions. Since the nucleation of a (polar) cluster is a rare process, it is often observed that in finite systems only one polar cluster emerges and as it cruises the system, collects quickly particles that are still in a gas phase. This leads, again for finite systems, to the emerge of global orientation order, i.e. global collective motion.

\section{FINAL REMARKS}

The study of this simple model has allowed us to address fundamental questions in the context of phase transitions in nonequilibrium systems. Note that the system becomes an equilibrium system only in the limit of $v_{0}=0$ and for reciprocal interactions, i.e. $\gamma=R_{0}$; otherwise the system is out of (thermodynamic) equilibrium. In this limit, a Cahn-Hilliard type of neutral phase separation is expected. By making $v_{0}>0$, and using reciprocal interactions, the system can also, not surprisingly, phase separate. For $\beta=0$, the spontaneous spin-flips ensure that the emerging clusters remain neutral with respect to their magnetization. Furthermore, these spontaneous, spin transitions render the motion of these aggregates diffusive, and thus, the phase separation is controlled at long time scales by diffusion, and a critical exponent of $-1 / 3$ is observed. On the other hand, with $\beta>0$ 
spin-flips are suppressed and for small enough $D$, clusters move ballistically in between interactions with other clusters. As result of this, the long-time dynamics of the phase separation process is very different with an asymptotic dynamics characterized by an exponent of $-2 / 3$. In summary, we have shown that there exist for reciprocal interactions two possible routes to neutral phase separation (NPS), which are characterized by two distinct critical exponents.

For nonreciprocal interactions, i.e. $\gamma<R_{0}$, the physics of the problem is dramatically affected. Notably, it is now possible to observe the emergence of polarized clusters. Moreover, we observe that clusters with the same sign of magnetization (or polarization) start to aggregate as the move ballistically at a speed that is roughly the active speed $v_{0}$. This process leads to asymptotic emergence of two spatially localized flocks moving in opposite directions. We call to this process polar phase separation (PPS) as oppose to NPS. In finite systems, this process can lead to the emergence of a single macroscopic cluster (or condensate), which implies the emergence - only possible for finite size sizes - of global polar order.

The fundamental lesson from our study is to prove that it is possible to conceive a microscopic model with no predefined symmetry at the level of the interactions moreover, without even possessing spin-spin interactions as in the classical or active Ising model - where (local) order emerges from the dynamical properties of the system and as direct consequence of breaking the action-reaction symmetry. Furthermore, we have shown that in the absence of the action-reaction symmetry, there is an intimate link between phase separation and the formation of highly-coherent, spatially localized moving flocks (i.e. collective motion). In summary, the study proves the existence of two fundamentally different types of active phase separation, NPS and PPS. In addition, we have shown that NPS is subdivided in two classes (characterized by exponents $-1 / 3$ and $-2 / 3$ ). These results are of key importance to understand that in Active Matter: i) there exist several phase-separation classes and ii) the spontaneous emergence of polar, self-organized patterns (i.e. flocks) does not require the minimization of a nonequilibrium version of a free energy that depends on the local "magnetization", but results from a pure dynamical process.

Acknowledgement LB acknowledges financial support from CONICET through Programa Houssay and PIP 11220110100794 and FP from Agence Nationale de la Recherche via Grant ANR-15-CE30-0002-01. Simulations were carried out in CRIMSON and CICADA clusters belonging to OCA and UNSA, respectively.
[1] T. Vicsek and A. Zafeiris, Physics Reports 517, 71 (2012).

[2] M. C. Marchetti, J. F. Joanny, S. Ramaswamy, T. B. Liverpool, M. R. J. Prost, and R. A. Simha, Rev. Mod. Phys. 85, 1143 (2013).

[3] M. Ballerini, N. Cabibbo, R. Candelier, A. Cavagna, E. Cisbani, I. Giardina, V. Lecomte, A. Orlandi, G. Parisi, A. Procaccini, et al., Proc. Natl. Acad. Sci. USA 105, 1232 (2008).

[4] J. Gautrais, F. Ginelli, R. Fournier, S. Blanco, M. Soria, H. Chaté, and G. Theraulaz, PLoS Comput Biol 8(9), e1002678 (2012).

[5] F. Ginelli, F. Peruani, M.-H. Pillot, H. Chaté, G. Theraulaz, and R. Bon, Proc. Natl. Acad. Sci. USA 112, 12729 (2015).

[6] S. Toulet, J. Gautrais, R. Bon, and F. Peruani, PLoS ONE 10, e0140188 (2015).

[7] D. Grossman, I. Aranson, and E. Ben-Jacob, New J. Phys. 10, 023036 (2008).

[8] J. Deseigne, O. Dauchot, and H. Chaté, Phys. Rev. Lett. 105, 098001 (2010).

[9] C. Weber, T. Hanke, J. Deseigne, S. Léonard, O. Dautchot, E. Frey, and H. Chaté, Phys. Rev. Lett. 110, 208001 (2013).

[10] K.-D. N. T. Lam, M. Schindler, and O. Dauchot, New J. Phys. 17, 113056 (2015).

[11] T. Vicsek, A. Czirok, E. B. Jacob, I. Cohen, and O. Shochet, Phys. Rev. Lett. 75, 1226 (1995).

[12] J. Toner and Y. Tu, Phys. Rev. Lett. 75, 4326 (1995).

[13] J. Toner and Y. Tu, Phys. Rev. E 58, 4828 (1998).

[14] O. O'Loan and M. Evans, Journal of Physics A: Mathematical and General 32, L99 (1999).

[15] A. Czirók, A.-L. Barabási, and T. Vicsek, Physical Review Letters 82, 209 (1999).

[16] A. P. Solon and J. Tailleur, Phys. Rev. Lett. 111, 078101 (2013).

[17] D. Strömbom, Journal of Theoretical Biology 283, 145 (2011).

[18] M. Moussaid, D. Helbing, and G. Theraulaz, 108, 6884 (2011).

[19] E. Ferrante, A. E. Turgut, M. Dorigo, and C. Huepe, Phys. Rev. Lett. 111, 268302 (2013).

[20] C. Huepe, E. Ferrante, T. Wenseleers, and A. Turgut, J. Stat. Phys. 158, 549 (2015).

[21] R. Soto and R. Golestanian, Phys. Rev. Lett. 112, 068301 (2014).

[22] L. Barberis and F. Peruani, Phys. Rev. Lett. 117, 248001 (2016).

[23] F. Peruani, J. Phys. Soc. Jpn. 86, 101010 (2017).

[24] H. Chaté, F. Ginelli, G. Grégoire, and F. Raynaud, Phys. Rev. E 77, 046113 (2008).

[25] J. Toner, Phys. Rev. E 86, 031918 (2012).

[26] S. Ramaswamy, R. A. Simha, and J. Toner, Europhys. Lett. 62, 196 (2003).

[27] H. Chaté, F. Ginelli, and R. Montagne, Phys. Rev. Lett. 96, 180602 (2006).

[28] S. Ngo, A. Peshkov, I. S. Aranson, E. Bertin, F. Ginelli, and H. Chaté, Phys. Rev. Lett. 113, 038302 (2014).

[29] F. Peruani, A. Deutsch, and M. Bär, Phys. Rev. E 74, 030904(R) (2006).

[30] F. Peruani, A. Deutsch, and M. Bär, Eur. Phys. J. Special 
Topics 157, 111 (2008).

[31] A. Baskaran and M. C. Marchetti, Phys. Rev. Lett. 101, 268101 (2008).

[32] F. Ginelli, F. Peruani, M. Bär, and H. Chaté, Phys. Rev. Lett. 104, 184502 (2010).

[33] A. Peshkov, I. S. Aranson, E. Bertin, H. Chaté, and F. Ginelli, Phys. Rev. Lett. 109, 268701 (2012).

[34] M. Abkenar, K. Marx, T. Auth, and G. Gompper, Phys. Rev. E 88, 062314 (2013).

[35] S. Weitz, A. Deutsch, and F. Peruani, Phys. Rev. E 92, 012322 (2015).

[36] R. J. Glauber, Journal of mathematical physics 4, 294 (1963).

[37] R. A. Blythe and M. R. Evans, J. Phys. A 40, R333 (2007).

[38] M. Cates and J. Tailleur, Phys. Rev. Lett. 100, 218103
(2008).

[39] F. Peruani, T. Klauss, A. Deutsch, and A. Voss-Boehme, Phys. Rev. Lett. 106, 128101 (2011).

[40] P. Romanczuk, I. Couzin, and L. Schimansky-Geier, Phys. Rev. Lett. 102, 010602 (2009).

[41] R. Grossmann, L. Schimansky-Geier, and P. Romanczuk, New Journal of Physics 15, 085014 (2013).

[42] An exception are active models with microscopic polar alignment and asymmetric interactions, where by, e.g., ignoring particles situated in front and moving in the opposite direction, can generate nematic order [40, 41]

[43] Strictly speaking neutral clusters for $\beta>0$ can be ballistic, however its speed decreases with the size of the cluster. When clusters contain a macroscopic fraction of the system, then the cluster speed has a vanishing speed. 